\title{
Exact Analytical Solution for Two-Dimensional Heat Transfer Equation through a Packed Bed Reactor
}

\author{
Mohammed Wassef Abdulrahman \\ Rochester Institute of Technology \\ Dubai, UAE \\ mwacad@rit.edu
}

\begin{abstract}
Heat transfer through packed bed reactors with chemical reactions can play a crucial role in determining the performance of such systems. To explain the effects of temperatures profiles on packed bed reactors, an exact analytical model has been derived by using Hankel and Laplace transform methods. The analysis is based on a transient two-dimensional model of cylindrical coordinates in which the energy equation is solved to yield the transient parameters of a packed bed flow. The model has been used to investigate the axial, radial and time temperature profiles and the effects of superficial velocity on the analytical solution. Comparisons with the experimental results and previous works are presented and show reasonable agreement with the analytical results. The model can be used to derive simple operating maps that would help to identify the effects heat transfer parameters on axial, radial and time temperature profiles.
\end{abstract}

Keywords: Packed bed reactors; Hankel and Laplace transform; Temperature profile; Superficial velocity

\section{Introduction}

A packed bed catalytic reactor is an assembly of usually uniformly sized catalytic particles, which are randomly arranged and firmly held in position within a vessel or tube. When the fluid containing the reactants flows through the packed bed a variety of physical and chemical phenomena occur in the reactor. Due to enormous complexity of these phenomena an exact mathematical description of packed bed reactors is virtually impossible and simplified mathematical models in terms of averaged quantities have been developed for their description.

There are many studies in the literature about packed beds. In 1950, Bernard and Wilhelm [1] described radial dispersion in packed beds by a Fickian model. In 1953, Danckwerts [2] published his celebrated paper on residence time distribution in continuous contacting vessels, including chemical reactors, and thus provided methods for measuring axial dispersion rates. These important contributions has set the direction in which the mathematical modelling of packed bed reactors have been developing. Jong et al. [3] have modelled the natural gas conversion process within the reformer by both chemical reaction and heat transfer models. The models gave data of the temperature along the reformer reactor. Abdulrahman [4-8] has studied experimentally the hydrodynamics and heat transfer of a multiphase bubble column. He has found new empirical equations to describe hydrodynamics and heat transfer of the multiphase reactor. Bert Koning [9] has studied the heat and mass transfer of the tubular catalyst bed theoretically. He has resolved the observed discrepancy between the experimental heat transfer parameters under reacting and non-reacting conditions. Abdulrahman [10-11] has studied numerically the heat transfer of the multiphase bubble column reactors by using 2-D CFD simulations. He has considered the slurry inside the multiphase bubble column as a homogeneous mixture. Abdulrahman [12] has modelled analytically the steady-state heat transfer behaviour of a gas flowing through a packed bed under the constant wall temperature conditions. He has derived equations for the steady state temperature distributions by using separation of variables method.

Despite extensive investigation of packed bed reactors and efforts to mathematically model them, there is still a lack of fundamental justification of the conventionally used continuum models, which employ a Fick and Fourier type description for the mass and heat dispersion fluxes respectively. Moreover, there is experimental evidence that cannot be explained with the standard dispersion models and is even contradicting to this approach. In addition to the problems associated with the formal, mathematical description of packed bed reactors, mathematical treatment of the governing model equations should also be carefully addressed. Due to the strong non-linearity of the reaction rate expressions, an analytical solution of 
the system of differential/algebraic equations can rarely be obtained. Therefore, the focus is usually on the numerical, approximate, solution of the equations.

The present work is an analytical investigation of a heat transfer in a tubular packed bed reactor with chemical reaction, which have not been addressed before. The analysis is based on existing two dimensional modeling methods for packed bed reactors. The energy equation for the fluid in a tube is solved to yield the transient parameters of a packed bed flow. The model uses the Hankel and Laplace transform methods to analytically solve the partial differential energy equation in cylindrical coordinates.

\section{Theoretical Analysis}

In the analyses of this paper, it is assumed that only two spatial cylindrical coordinates, radial $(r)$ and axial $(z)$ are considered. The angular coordinate is neglected because of similarity. Also, it is assumed that fluid properties are considered constants, and the heat generated (or consumed) by chemical reactions is considered constant to keep the linearity feature of the energy partial differential equation. The most often heat balance used in 2-D model for constant effective conductivity is the pseudo-homogeneous model, which is given by equation:

$$
\frac{1}{P e_{l}} \frac{\partial^{2} \theta}{\partial \bar{z}^{2}}-\frac{\partial \theta}{\partial \bar{z}}+\frac{X}{P e_{r}} \frac{\partial^{2} \theta}{\partial \bar{r}^{2}}+\frac{X}{P e_{r}} \frac{1}{\bar{r}} \frac{\partial \theta}{\partial \bar{r}}-Q_{i}=\frac{\partial \theta}{\partial \bar{t}}
$$

where, $P e_{l}=\frac{u_{s} L}{\alpha_{a x}} \quad, \quad P e_{r}=\frac{u_{s} R}{\alpha_{r}} \quad, \quad Q_{i}=\frac{\rho C_{p} L}{u_{s}} R_{i} \quad, \quad X=\frac{L}{R} \quad, \quad R_{i}=-\frac{R_{T}}{\Delta H_{r}}$

and the dimensionless numbers and variables are defined as:

$$
\theta=\frac{T-T_{w}}{T_{0}-T_{w}}=\frac{T-T_{w}}{\Delta T} \quad, \quad \bar{z}=\frac{z}{L} \quad, \quad \bar{r}=\frac{r}{R} \quad, \quad \bar{t}=\frac{u_{s} t}{L}
$$

The following boundary and initial conditions are applied:

$$
\begin{gathered}
\left.\theta\right|_{\bar{z}=0}=1,\left.\quad \frac{\partial \theta}{\partial \bar{z}}\right|_{\bar{z}=\infty}=0,\left.\quad \theta\right|_{\bar{t}=0}=\theta_{i},\left.\quad \frac{\partial \theta}{\partial \bar{r}}\right|_{\bar{r}=0}=0 \\
\left.\frac{\partial \theta}{\partial \bar{r}}\right|_{\bar{r}=1}=-\frac{h_{w}}{K_{e r}} \theta
\end{gathered}
$$

To solve Eq. (1) analytically, it will be assumed that the volumetric heat generation $R_{T}$ and the volumetric production (consumption) of component $R_{i}$ are constant. The general form of finite Hankel transform of order $v$ of a function $f(r)$ that is associated with a general boundary condition of:

$$
f^{\prime}(r)+h f(r)=0 \quad \text { at } r=1
$$

is defined by [13]:

$$
\mathcal{H}_{v}\{f(r)\}=F_{v}\left(\lambda_{n}\right)=\int_{0}^{R} r f(r) J_{v}\left(\lambda_{n} r\right) d r
$$

where $\lambda_{n}$ are the roots of the equation:

$$
\lambda_{n} J_{v}^{\prime}\left(\lambda_{n} R\right)+h J_{v}\left(\lambda_{n} R\right)=0
$$


The corresponding inverse transform of Eq. (6) is given by:

$$
f(r)=\mathcal{H}_{v}^{-1}\left\{F_{v}\left(\lambda_{n}\right)\right\}=2 \sum_{n=1}^{\infty} \frac{\lambda_{n}^{2} F_{v}\left(\lambda_{n}\right) J_{v}\left(\lambda_{n} r\right)}{\left\{\left(\lambda_{n}^{2}+h^{2}\right) R^{2}-v^{2}\right\} J_{v}^{2}\left(\lambda_{n} R\right)}
$$

This finite Hankel transform has the following operational property:

$$
\mathcal{H}_{v}\left\{\frac{d^{2} f(r)}{d r^{2}}+\frac{1}{r} \frac{d f(r)}{d r}-\left(\frac{v}{r}\right)^{2} f(r)\right\}=-\lambda_{n}^{2} F_{v}\left(\lambda_{n}\right)+R\left[f^{\prime}(r)+h f(r)\right] J_{v}\left(\lambda_{n} R\right)
$$

which is, by using Eq. (7) will be:

$$
\mathcal{H}_{v}\left\{\frac{d^{2} f(r)}{d r^{2}}+\frac{1}{r} \frac{d f(r)}{d r}-\left(\frac{v}{r}\right)^{2} f(r)\right\}=-\lambda_{n}^{2} F_{v}\left(\lambda_{n}\right)-\frac{\lambda_{n} R}{h}\left[f^{\prime}(r)+h f(r)\right] J_{v}^{\prime}\left(\lambda_{n} R\right)
$$

Thus result (10) involves $f^{\prime}(r)+h f(r)=0$ as the boundary condition. For zero-order, Eq. (9) can be written as:

$$
\mathcal{H}_{0}\left\{\frac{d^{2} f(r)}{d r^{2}}+\frac{1}{r} \frac{d f(r)}{d r}\right\}=-\lambda_{n}^{2} F_{0}\left(\lambda_{n}\right)+R\left[f^{\prime}(r)+h f(r)\right] J_{0}\left(\lambda_{n} R\right)
$$

The aforementioned two-dimensional heat balance in cylindrical coordinates and its associated initial and boundary conditions are analytically solved by successive implementation of the finite Hankel transform (Eqs.(6)-(11)) and the Laplace transform. By comparing Eqs. (4) and (5), it is found that; $f(r)=\theta(\bar{r}, \bar{z}, \bar{t})$ and,

$$
h=\frac{h_{w}}{k_{e r}}
$$

First, utilizing the second kind of finite Hankel transform (Eq. (11)) in Eq. (1) with respect to ( $r$ ) results in:

$$
\frac{1}{P e_{l}} \frac{\partial^{2} \theta_{H}}{\partial \bar{z}^{2}}-\frac{\partial \theta_{H}}{\partial \bar{z}}-\frac{X}{P e_{r}} \lambda_{n}^{2} \theta_{H}-Q_{i} \frac{J_{1}\left(\lambda_{n}\right)}{\lambda_{n}}=\frac{\partial \theta_{H}}{\partial \bar{t}}
$$

where,

$\lambda_{n}$ : Finite Hankel transform parameter as determined by the transcendental Eq. (7).

$J_{0}\left(\lambda_{n}\right)$ : Zero-order Bessel function of the first kind.

$J_{1}\left(\lambda_{n}\right)$ : First-order Bessel function of the first kind.

$\theta_{H}\left(\lambda_{n}, \bar{z}, \bar{t}\right)$ : Second kind of finite Hankel transform for $\theta(\bar{r}, \bar{z}, \bar{t})$ as defined by the following conjugate equations:

$$
\begin{gathered}
\theta_{H}\left(\lambda_{n}, \bar{z}, \bar{t}\right)=\mathcal{H}\{\theta(\bar{r}, \bar{z}, \bar{t})\}=\int_{0}^{1} \bar{r} \theta(\bar{r}, \bar{z}, \bar{t}) J_{0}\left(\lambda_{n} \bar{r}\right) d \bar{r} \\
\theta(\bar{r}, \bar{z}, \bar{t})=\mathcal{H}_{0}^{-1}\left\{\theta_{H}\left(\lambda_{n}, \bar{z}, \bar{t}\right)\right\}=2 \sum_{n=1}^{\infty} \frac{\lambda_{n}^{2} \theta_{H}\left(\lambda_{n}, \bar{z}, \bar{t}\right)}{\left(\lambda_{n}^{2}+h^{2}\right)} \frac{J_{0}\left(\lambda_{n} \bar{r}\right)}{\left|J_{0}\left(\lambda_{n}\right)\right|^{2}}
\end{gathered}
$$

Accordingly, the initial and boundary conditions after the finite Hankel transform are:

$$
\theta_{H}\left(\lambda_{n}, \bar{z}=0, \bar{t}\right)=\frac{J_{1}\left(\lambda_{n}\right)}{\lambda_{n}}
$$




$$
\begin{gathered}
\frac{\partial \theta_{H}\left(\lambda_{n}, \bar{z}=\infty, \bar{t}\right)}{\partial \bar{z}}=0 \\
\theta_{H}\left(\lambda_{n}, \bar{z}, \bar{t}=0\right)=\frac{J_{1}\left(\lambda_{n}\right)}{\lambda_{n}} \theta_{i}
\end{gathered}
$$

By applying the Laplace transform with respect to $t$ to Eq. (13):

$$
\frac{1}{P e_{l}} \frac{\partial^{2} \theta_{H L}}{\partial \bar{z}^{2}}-\frac{\partial \theta_{H L}}{\partial \bar{z}}-\left(\frac{X}{P e_{r}} \lambda_{n}^{2}+s\right) \theta_{H L}-Q_{i} \frac{J_{1}\left(\lambda_{n}\right)}{\lambda_{n}} \frac{1}{s}+\theta_{i}=0
$$

where $(s)$ denotes to the Laplace transform parameter and $\theta_{H L}\left(\lambda_{n}, \bar{z}, s\right)$ represents the Laplace transform of $\theta_{H}\left(\lambda_{n}, \bar{z}, \bar{t}\right)$. As a consequence, the boundary conditions after the Laplace transform become:

$$
\begin{gathered}
\theta_{H L}\left(\lambda_{n}, \bar{z}=0, s\right)=\frac{J_{1}\left(\lambda_{n}\right)}{\lambda_{n} s} \\
\frac{\partial \theta_{H L}\left(\lambda_{n}, \bar{z}=\infty, s\right)}{\partial \bar{z}}=0
\end{gathered}
$$

Equation (19) is a non-homogeneous ordinary differential equation. The general solution consists of both, complementary and particular solutions, which is:

$$
\begin{gathered}
\theta_{H L}= \\
a_{1} \exp \left(\frac{P e_{L} \bar{z}}{2}\right) \exp \left(\sqrt{P e_{L}} \bar{z} \sqrt{\frac{P e_{L}}{4}+\frac{X}{P e_{r}} \lambda_{n}^{2}+s}\right)+ \\
a_{2} \exp \left(\frac{P e_{L} \bar{z}}{2}\right) \exp \left(-\sqrt{P e_{L}} \bar{z} \sqrt{\frac{P e_{L}}{4}+\frac{X}{P e_{r}} \lambda_{n}^{2}+s}\right)+\frac{\theta_{i}}{s+\frac{X \lambda_{n}^{2}}{P e_{r}}}-\frac{\frac{Q_{i} J_{1}\left(\lambda_{n}\right)}{\lambda_{n}}}{s^{2}+\frac{X \lambda_{n}^{2}}{P e_{r}} S}
\end{gathered}
$$

where $a_{1}$ and $a_{2}$ are two unknown coefficients which need to be determined. $a_{1}$ and $a_{2}$ in Eq. (22) can be straightforwardly determined with the aid of the boundary conditions found with Eqs. (20) and (21):

$$
\begin{gathered}
a_{1}=0 \\
a_{2}=\frac{J_{1}\left(\lambda_{n}\right)}{\lambda_{n} s}-\frac{\theta_{i}}{\frac{X \lambda_{n}^{2}}{P e_{r}}+s}+\frac{\frac{Q_{i} J_{1}\left(\lambda_{n}\right)}{\lambda_{n}}}{s^{2}+\frac{X \lambda_{n}^{2}}{P e_{r}} s}
\end{gathered}
$$

Introducing $a_{1}$ and $a_{2}$ (Eqs. (23) and (24)) into Eq. (22), the general solution can be expressed as:

$$
\begin{aligned}
\theta_{H L}=\left(\frac{J_{1}\left(\lambda_{n}\right)}{\lambda_{n} s}-\frac{\theta_{i}}{\frac{X \lambda_{n}^{2}}{P e_{r}}+s}+\frac{\frac{Q_{i} J_{1}\left(\lambda_{n}\right)}{\lambda_{n}}}{s^{2}+\frac{X \lambda_{n}^{2}}{P e_{r}} s}\right) \exp \left(\frac{P e_{L} \bar{z}}{2}\right) \exp \left(-\sqrt{P e_{L}} \bar{z} \sqrt{\frac{P e_{L}}{4}+\frac{X}{P e_{r}} \lambda_{n}^{2}+s}\right)+\frac{\theta_{i}}{s+\frac{X \lambda_{n}^{2}}{P e_{r}}}- \\
\\
\frac{\frac{Q_{i} J_{1}\left(\lambda_{n}\right)}{\lambda_{n}}}{s^{2}+\frac{X \lambda_{n}^{2}}{P e_{r}} s}
\end{aligned}
$$


The solution for original domain $\theta(\bar{r}, \bar{z}, \bar{t})$ is the consecutive inversion of the Laplace transform and the finite Hankel transform of $\theta_{H L}\left(\lambda_{n}, \bar{z}, s\right)$. For convenience, the Laplace inversion is performed first. The Laplace inverse transform for Eq. (25) is obtained using the s-shift theorem and the following Laplace inverse formula [14]:

$$
L^{-1}\left[\frac{e^{-\alpha \sqrt{s}}}{s-A^{2}}\right]=\frac{1}{2}\left[\exp \left(A^{2} t-A \alpha\right) \operatorname{erfc}\left(\frac{\alpha}{2 \sqrt{t}}-A \sqrt{t}\right)+\exp \left(A^{2} t+A \alpha\right) \operatorname{erfc}\left(\frac{\alpha}{2 \sqrt{t}}+A \sqrt{t}\right)\right]
$$

By applying inverse Laplace transform, Eq. (25) can be written as:

$$
\begin{gathered}
\theta_{H}\left(\lambda_{n}, \bar{z}, \bar{t}\right)=L^{-1}\left[\theta_{H L}\left(\lambda_{n}, \bar{z}, s\right)\right]=L^{-1}\left[\frac{J_{1}\left(\lambda_{n}\right)}{\lambda_{n}} \exp \left(\frac{P e_{L} \bar{z}}{2}\right) \frac{1}{s} \exp \left(-\sqrt{P e_{L}} \bar{z} \sqrt{\left.\frac{P e_{L}}{4}+\frac{X}{P e_{r}} \lambda_{n}^{2}+s\right)}\right)-\right. \\
L^{-1}\left[\frac{\theta_{i}}{\frac{X \lambda_{n}^{2}}{P e_{r}}+s} \exp \left(\frac{P e_{L} \bar{z}}{2}\right) \exp \left(-\sqrt{P e_{L}} \bar{z} \sqrt{\left.\frac{P e_{L}}{4}+\frac{X}{P e_{r}} \lambda_{n}^{2}+s\right)}\right)+\right. \\
L^{-1}\left[\frac{\frac{Q_{i} J_{1}\left(\lambda_{n}\right)}{\lambda_{n}}}{s^{2}+\frac{X \lambda_{n}^{2}}{P e_{r}} s} \exp \left(\frac{P e_{L} \bar{z}}{2}\right) \exp \left(-\sqrt{P e_{L}} \bar{z} \sqrt{\frac{P e_{L}}{4}+\frac{X}{P e_{r}} \lambda_{n}^{2}+s}\right)\right]+L^{-1}\left[\frac{\theta_{i}}{s+\frac{X \lambda_{n}^{2}}{P e_{r}}}\right]-L^{-1}\left[\frac{\frac{Q_{i} J_{1}\left(\lambda_{n}\right)}{\lambda_{n}}}{s^{2}+\frac{X \lambda_{n}^{2}}{P e_{r}} s}\right]
\end{gathered}
$$

Eq. (27) consists of five terms that they have to be transformed inversely by using inverse Laplace transform. By using the s-shift theorem and the Laplace inverse formula Eq. (26) for the first and second terms of Eq. (27), and by using partial fraction expansion method and the Laplace inverse formula Eq. (26) in the third term. By completing the square in the denominator and using partial fraction expansion method in the fifth term, the total solution of inverse Laplace transform of Eq. (27):

$$
\begin{gathered}
\theta_{H}\left(\lambda_{n}, \bar{z}, \bar{t}\right)=L^{-1}\left[\theta_{H L}\left(\lambda_{n}, \bar{z}, s\right)\right]=\left\{\frac { J _ { 1 } ( \lambda _ { n } ) } { 2 \lambda _ { n } } \operatorname { e x p } ( \frac { P e _ { L } \overline { z } } { 2 } - A ^ { 2 } \overline { t } ) \left[\exp \left(A^{2} \bar{t}-A \alpha\right) \operatorname{erfc}\left(\frac{\alpha}{2 \sqrt{\bar{t}}}-A \sqrt{\bar{t}}\right)+\right.\right. \\
\left.\left.\exp \left(A^{2} \bar{t}+A \alpha\right) \operatorname{erfc}\left(\frac{\alpha}{2 \sqrt{\bar{t}}}+A \sqrt{\bar{t}}\right)\right]\right\}-\left\{\frac { \theta _ { i } } { 2 } \operatorname { e x p } ( \frac { P e _ { L } \overline { z } } { 2 } - A ^ { 2 } \overline { t } ) \left[\exp \left(B^{2} \bar{t}-B \alpha\right) \operatorname{erfc}\left(\frac{\alpha}{2 \sqrt{\bar{t}}}-B \sqrt{\bar{t}}\right)+\right.\right. \\
\left.\left.\exp \left(B^{2} \bar{t}+B \alpha\right) \operatorname{erfc}\left(\frac{\alpha}{2 \sqrt{\bar{t}}}+B \sqrt{\bar{t}}\right)\right]\right\}+\left\{\frac { Q _ { i } J _ { 1 } ( \lambda _ { n } ) } { \lambda _ { n } } \frac { P e _ { r } } { 2 X \lambda _ { n } ^ { 2 } } \operatorname { e x p } ( \frac { P e _ { L } \overline { z } } { 2 } - A ^ { 2 } \overline { t } ) \left[\operatorname { e x p } \left(B^{2}+\frac{X \lambda_{n}^{2}}{P e_{r}}-\right.\right.\right. \\
\left.\sqrt{P e_{L}} A \bar{z}\right) \operatorname{erfc}\left(\frac{\bar{z}}{2} \sqrt{\frac{P e_{L}}{\bar{t}}}-A \sqrt{\bar{t}}\right)+\exp \left(B^{2}+\frac{X \lambda_{n}^{2}}{P e_{r}} \bar{t}+\sqrt{P e_{L}} A \bar{z}\right) \operatorname{erfc}\left(\frac{\bar{z}}{2} \sqrt{\frac{P e_{L}}{\bar{t}}}+A \sqrt{\bar{t}}\right)+ \\
\left.\left.\exp \left(B^{2} \bar{t}-\sqrt{P e_{L}} B \bar{z}\right) \operatorname{erfc}\left(\frac{\bar{z}}{2} \sqrt{\frac{P e_{L}}{\bar{t}}}-B \sqrt{\bar{t}}\right)+\exp \left(B^{2} \bar{t}+\sqrt{P e_{L}} B \bar{z}\right) \operatorname{erfc}\left(\frac{\bar{z}}{2} \sqrt{\frac{P e_{L}}{\bar{t}}}+B \sqrt{\bar{t}}\right)\right]\right\}+ \\
\left\{\theta_{i} \exp \left(-\frac{X \lambda_{n}^{2}}{P e_{r}} \bar{t}\right)\right\}-\left\{\frac{Q_{i} J_{1}\left(\lambda_{n}\right)}{\lambda_{n}} \frac{P e_{r}}{X \lambda_{n}^{2}}\left[1-\exp \left(-\frac{X \lambda_{n}^{2}}{P e_{r}} \bar{t}\right)\right]\right\}
\end{gathered}
$$

where $\alpha=\sqrt{P e_{L}} \bar{Z} \quad, \quad A=\sqrt{\frac{P e_{L}}{4}+\frac{X}{P e_{r}} \lambda_{n}^{2}} \quad, \quad B=\sqrt{\frac{P e_{L}}{4}}$,

It should be noted that Eq. (28) is only valid for $\lambda_{n} \neq 0$. For $\lambda_{n}=0$, Eqs. (13), (16) and (18) give unlimited solution for temperature, so it is neglected. The next step is applying inverse Hankel transform to Eq. (28). The original domain solution can be straightforwardly obtained using the finite Hankel inversion of Eq. (15). To get the original domain 
solution, substitute Eqs. (12) and (28) into Eq. (15) to get $\theta(\bar{r}, \bar{z}, \bar{t})$. Temperature distribution $T(r, z, t)$ can be obtained by substituting Eq. (2) into equation of $\theta(\bar{r}, \bar{z}, \bar{t})$.

$$
\begin{aligned}
& T(r, z, t)=T_{w}+2 \Delta T \sum_{n=1}^{\infty} \frac{\lambda_{n}^{2}}{\left(\lambda_{n}^{2}+\left(\frac{h_{w}}{k_{e r}}\right)^{2}\right)} \frac{J_{0}\left(\lambda_{n}\left(\frac{r}{R}\right)\right)}{\left|J_{0}\left(\lambda_{n}\right)\right|^{2}} \|\left\{\frac { J _ { 1 } ( \lambda _ { n } ) } { 2 \lambda _ { n } } \operatorname { e x p } ( \frac { P e _ { L } Z } { 2 L } - A ^ { 2 } \frac { u _ { s } t } { L } ) \left[\operatorname { e x p } \left(A^{2} \frac{u_{s} t}{L}-\right.\right.\right. \\
& \left.\left.A \alpha) \operatorname{erfc}\left(\frac{\alpha}{2 \sqrt{\frac{u_{s} t}{L}}}-A \sqrt{\frac{u_{s} t}{L}}\right)+\exp \left(A^{2} \frac{u_{s} t}{L}+A \alpha\right) \operatorname{erfc}\left(\frac{\alpha}{2 \sqrt{\frac{u_{s} t}{L}}}+A \sqrt{\frac{u_{s} t}{L}}\right)\right]\right\}-\left\{\frac { \theta _ { i } } { 2 } \operatorname { e x p } \left(\frac{P e_{L} z}{2 L}-\right.\right. \\
& \left.A^{2} \frac{u_{s} t}{L}\right)\left[\exp \left(B^{2} \frac{u_{s} t}{L}-B \alpha\right) \operatorname{erfc}\left(\frac{\alpha}{2 \sqrt{\frac{u_{s} t}{L}}}-B \sqrt{\frac{u_{s} t}{L}}\right)+\exp \left(B^{2} \frac{u_{s} t}{L}+B \alpha\right) \operatorname{erfc}\left(\frac{\alpha}{2 \sqrt{\frac{u_{s} t}{L}}}+\right.\right. \\
& \left.\left.\left.B \sqrt{\frac{u_{s} t}{L}}\right)\right]\right\}+\left\{\frac { Q _ { i } J _ { 1 } ( \lambda _ { n } ) } { \lambda _ { n } } \frac { P e _ { r } } { 2 X \lambda _ { n } ^ { 2 } } \operatorname { e x p } ( \frac { P e _ { L } z } { 2 L } - A ^ { 2 } \frac { u _ { s } t } { L } ) \operatorname { e x p } ( B ^ { 2 } + \frac { X \lambda _ { n } ^ { 2 } } { P e _ { r } } \frac { u _ { s } t } { L } - \frac { \sqrt { P e _ { L } } A z } { L } ) \operatorname { e r f c } \left(\frac{z}{2 L} \sqrt{\frac{P e_{L}}{u_{S} t}}-\right.\right. \\
& \left.A \sqrt{\frac{u_{s} t}{L}}\right)+\exp \left(B^{2}+\frac{X \lambda_{n}^{2}}{P e_{r}} \frac{u_{s} t}{L}+\frac{\sqrt{P e_{L}} A z}{L}\right) \operatorname{erfc}\left(\frac{z}{2 L} \sqrt{\frac{P e_{L}}{\frac{u_{S} t}{L}}}+A \sqrt{\frac{u_{s} t}{L}}\right)+ \\
& \exp \left(B^{2} \frac{u_{s} t}{L}-\sqrt{P e_{L}} B \bar{z}\right) \operatorname{erfc}\left(\frac{z}{2 L} \sqrt{\frac{P e_{L}}{\frac{u_{S} t}{L}}}-B \sqrt{\frac{u_{s} t}{L}}\right)+\exp \left(B^{2} \frac{u_{s} t}{L}+\frac{\sqrt{P e_{L}} A z}{L}\right) \operatorname{erfc}\left(\frac{z}{2 L} \sqrt{\frac{P e_{L}}{\frac{u_{S} t}{L}}}+\right. \\
& \left.\left.B \sqrt{\frac{u_{s} t}{L}}\right)\right\}+\left\{\theta_{i} \exp \left(-\frac{X \lambda_{n}^{2}}{P e_{r}} \frac{u_{s} t}{L}\right)\right\}-\left\{\frac{Q_{i} J_{1}\left(\lambda_{n}\right)}{\lambda_{n}} \frac{P e_{r}}{X \lambda_{n}^{2}}\left[1-\exp \left(-\frac{X \lambda_{n}^{2}}{P e_{r}} \frac{u_{s} t}{L}\right)\right]\right\} \rrbracket
\end{aligned}
$$

\subsection{Effective radial thermal conductivity}

The correlation of effective radial thermal conductivity $K_{e r}$, proposed by Bauer and Schlunder [15, 16] lumps conduction through the solid and the fluid, as well as heat radiation:

$$
\begin{gathered}
\frac{K_{e r}}{K_{f}}=\frac{\rho u_{s} C_{p}}{K_{f}} \frac{F d_{p v}}{8\left[2-\left(1-2 \frac{d_{p v}}{d_{t}}\right)^{2}\right]}+(1-\sqrt{1-\varepsilon})\left(1+\varepsilon\left(2.27 \times 10^{-7} \frac{e}{2-e} T^{3} \frac{d_{p v}}{K_{f}}\right)\right)+\sqrt{1-\varepsilon}\left(\frac{K_{r s}}{K_{f}}\right) \\
\left.\frac{K_{r s}}{K_{f}}=\frac{2}{\left(\frac{K_{S}+K_{r}-C\left(\frac{1-\varepsilon}{\varepsilon}\right)^{\frac{10}{9}}}{K_{S}}\right)}\right)\left(\frac{C\left(\frac{1-\varepsilon}{\varepsilon}\right)^{\frac{10}{9}}\left(\frac{K_{\text {solid }}}{K_{f}}+\frac{K_{r a d}}{K_{f}}-1\right)}{\left(\frac{K_{S}+K_{r}-C\left(\frac{1-\varepsilon}{\varepsilon}\right)^{\frac{10}{9}}}{K_{S}}\right)^{2}} \ln \left(\frac{\frac{K_{\text {solid }}}{K_{f}}+\frac{K_{r a d}}{K_{f}}}{C\left(\frac{1-\varepsilon}{\varepsilon}\right)^{\frac{10}{9}}}\right)+\frac{C\left(\frac{1-\varepsilon}{\varepsilon}\right)^{\frac{10}{9}}+1}{2 C\left(\frac{1-\varepsilon}{\varepsilon}\right)^{\frac{10}{9}}}\left(K_{r}-C\left(\frac{1-\varepsilon}{\varepsilon}\right)^{\frac{10}{9}}\right)-\right. \\
\left.\frac{C\left(\frac{1-\varepsilon}{\varepsilon}\right)^{\frac{10}{9}}-1}{\left(\frac{K_{S}+K_{r}-C\left(\frac{1-\varepsilon}{\varepsilon}\right)^{\frac{10}{9}}}{K_{S}}\right)}\right]
\end{gathered}
$$


where, $F=\left\{\begin{array}{cl}1.15 & \text { for spherical particles } \\ 1.75 & \text { for cylindrical particles }\end{array}\right.$

$C=\left\{\begin{aligned} 1.25 & \text { for spherical particles } \\ 2.5 & \text { for cylindrical particles }\end{aligned}\right.$

\subsection{Effective axial thermal conductivity}

Votruba et al. [17] have obtained the following empirical correlation for the Peclet number for heat dispersion in axial direction for packing of spheres and rings of different materials:

$$
\frac{1}{P e_{h a x}}=\frac{K_{e a x}}{u_{s} \rho C_{p} d_{p v}}=\frac{K_{a x 0}}{K_{f}}\left(P e_{h 0}\right)^{-1}+\frac{14.5}{d_{p}\left(1+C(R e P r)^{-1}\right)} \quad(R e<1000)
$$

In this equation, $C$ is a constant depending on the properties of the solid and of the aspect ratio and has a value between 0 and 5 .

\subsection{Wall heat transfer coefficient}

As in case of the effective radial and axial thermal conductivity, the wall heat transfer coefficient is commonly defined as the sum of a flow-dependent and a flow-independent heat transfer coefficient; $h_{w}=h_{w 0}+h_{w f}$. In correlations, $h_{w}$ is usually expressed in the form of a dimensionless Nusselt number $N u ; N u_{w}=\frac{h_{w} d_{p v}}{K_{f}}$. Expressions for the contributions of convection usually have the form; $N u_{w f}=\frac{h_{w f} d_{p v}}{K_{f}}=C(R e)^{n_{1}}(P r)^{n_{2}}$, in which $C$ is a constant depending on the aspect ratio. In this paper, the correlation of Martin and Nilles (1993) is used, where; $N u_{w f}=0.19(\operatorname{Re})^{0.75}(P r)^{-0.42}$ with (using data of Borkink, 1991), where; $N u_{w 0}=\frac{h_{w 0} d_{p v}}{K_{f}}=\left(1.3+\frac{5}{N} \frac{K_{e r}}{K_{f}}\right)$

\section{Results}

The axial temperature distribution of this work is compared with other published works. Figure 1 shows the comparison of this work with the work of Obara [18]. Obara used finite differences method for his analysis. The analytical solution shows good agreement with Obara's work for the three different inlet temperatures. The maximum percentage difference between the two temperature profiles is $7.5 \%$.

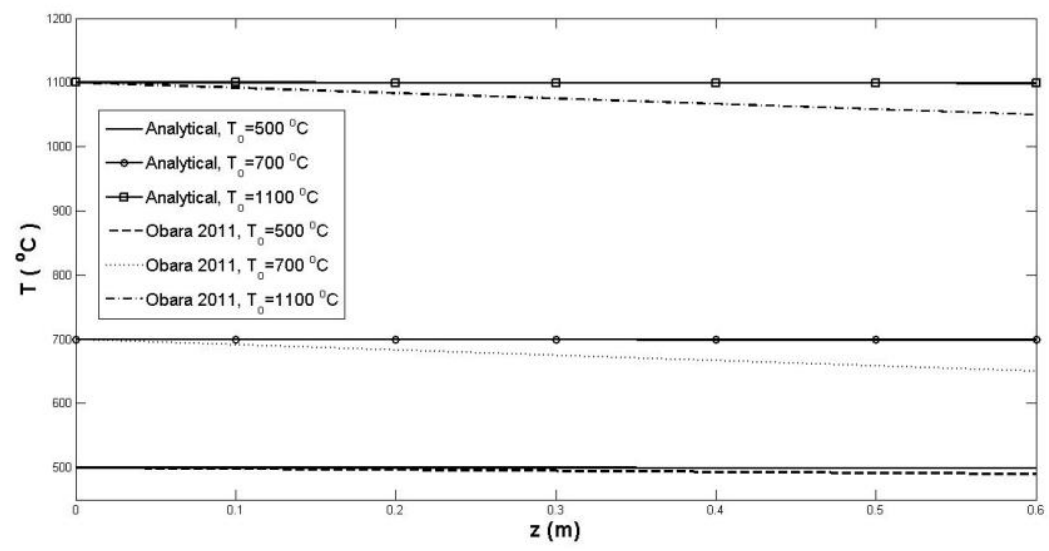

Fig. 1: Comparison of the analytical solution with the published numerical analysis. 
The results of this work is also compared with the experimental work of Yutaka et.al [19]. Figure 2 shows the axial temperature distribution for the velocity of $0.83 \mathrm{~m} / \mathrm{s}$ with the maximum percentage error of $3 \%$.

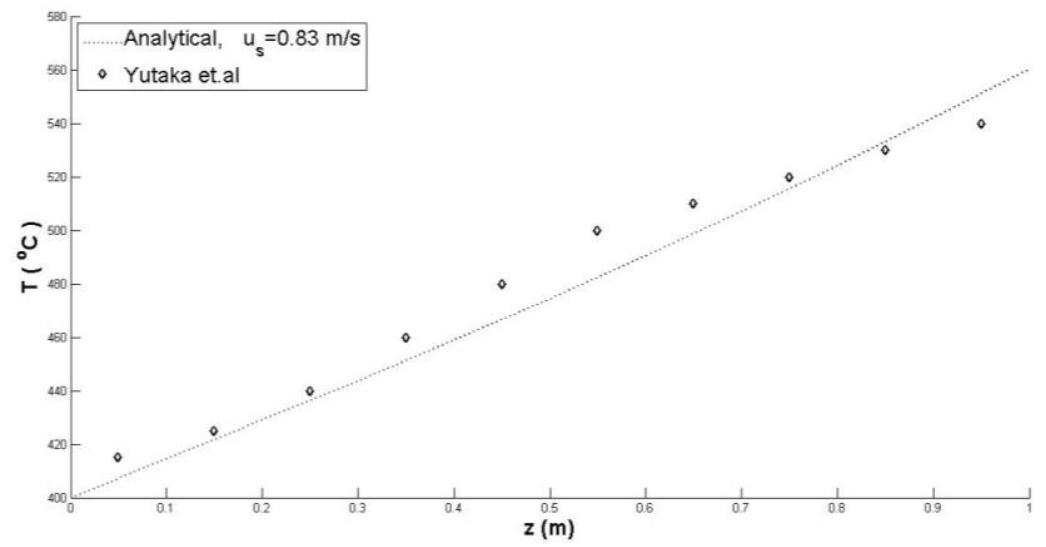

Fig. 2: Comparison of the axial temperature profile of analytical solution with Yutaka et.al experiment.

The time temperature distribution of this work is compared with another published work. Figure 3 shows the comparison of this work with the work of D. Wen and Y. Ding [20]. The analytical solution shows good agreement with Wen's work. The maximum percentage difference between the two temperature profiles is $8.3 \%$.

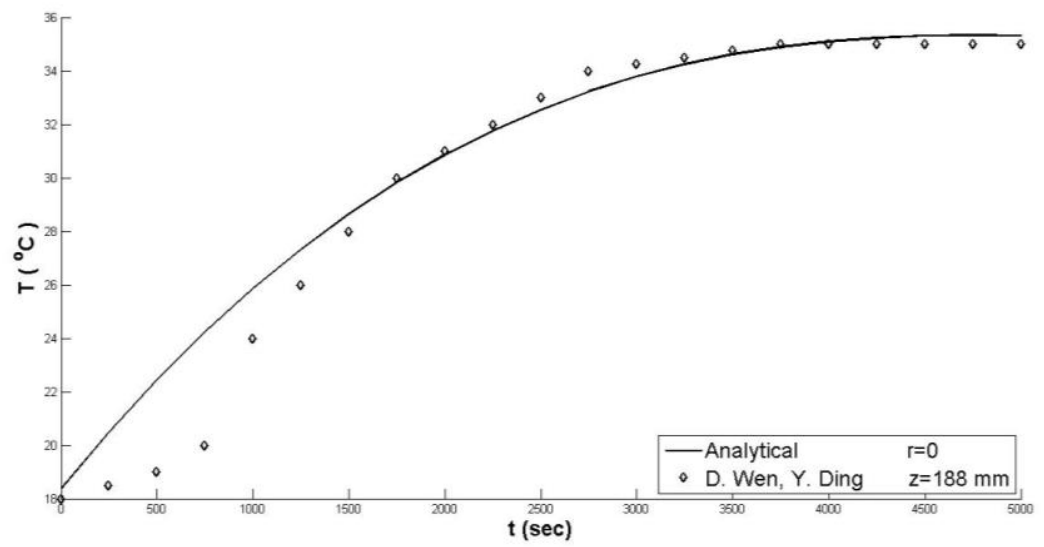

Fig. 3: Comparison of the time temperature profile of analytical solution with Wen et.al.

\section{Conclusion}

An analytical two dimensional model was developed, based on solution to the energy equation and, expression for the temperature was derived for tubular packed bed flow. By comparing the theoretical results with the experimental, it was demonstrated that the two dimensional analytical model (by Hankel and Laplace transform), is capable of describing the transient behaviour of radial and axial temperature profiles. The model of this work can be used for many types of flow that have heat transfer with or without chemical reactions. By using the equation of temperature that is derived in this analysis, one can easily study the effect of all parameters on the temperature profiles to know the effect of these parameters on heat transfer. 


\section{Nomenclature}

\begin{tabular}{|c|l|c|l|}
\hline Symbol & \multicolumn{1}{|c|}{ Definition } & Symbol & \multicolumn{1}{|c|}{ Definition } \\
\hline$u_{s}$ & Superficial velocity $(\mathrm{m} / \mathrm{s})$ & $R_{t}$ & Tube radius $(\mathrm{m})$ \\
$\rho$ & Fluid density $\left(\mathrm{Kg} / \mathrm{m}^{3}\right)$ & $\Delta H_{r}$ & Reaction enthalpy $(\mathrm{J} / \mathrm{mole})$ \\
$C_{p}$ & Heat capacity $(\mathrm{J} / \mathrm{Kg} . \mathrm{K})$ & $R_{i}$ & Reaction rate $(\mathrm{mole} / \mathrm{Kg} . \mathrm{s})$ \\
$T$ & Temperature $\left({ }^{\circ} \mathrm{C}\right)$ & $d_{p v}$ & Volume equivalent sphere diameter $(\mathrm{m})$ \\
$T_{0}$ & Inlet temperature $\left({ }^{\circ} \mathrm{C}\right)$ & $d_{t}$ & Tube diameter $(\mathrm{m})$ \\
$T_{i}$ & Initial temperature $\left({ }^{\circ} \mathrm{C}\right)$ & $\varepsilon$ & Porosity \\
$K_{e a x}$ & Effective axial fluid conductivity $\left(\mathrm{W} /{ }^{\circ} \mathrm{C} . \mathrm{K}\right)$ & $R e$ & Reynolds number \\
$K_{e r}$ & Effective radial fluid conductivity $\left(\mathrm{W} /{ }^{\circ} \mathrm{C} . \mathrm{K}\right)$ & $P r$ & Prandtle number \\
$R_{T}$ & Heat generation per reactor volume $\left(\mathrm{W} / \mathrm{m}^{3}\right)$ & $N u$ & Nusselt number \\
$h_{w}$ & Wall heat transfer coefficient $\left(\mathrm{W} / \mathrm{m}^{2} .{ }^{\circ} \mathrm{C}\right)$ & $P e_{e r}$ & Effective radial Peclet number \\
$T_{w}$ & Wall temperature $\left({ }^{\circ} \mathrm{C}\right)$ & $\alpha_{a x}$ & Axial thermal diffusivity \\
$T_{o}$ & Inlet temperature $\left({ }^{\circ} \mathrm{C}\right)$ & $\alpha_{r}$ & Radial thermal diffusivity \\
$L$ & Tube length $(\mathrm{m})$ & $e$ & Emissivity \\
\hline
\end{tabular}

\section{References}

[1] R. A. Bernard and R. H. Wilhelm, Chem. Eng. Progr. Symposium Ser. 46, 233, 1950

[2] P. V. Danckwerts, "Continuous flow systems. Distribution of residence times," Chem. Eng. Sci., vol. 1, pp. 1-13, 1953.

[3] M. De Jong, A. H. M. E. Reinders, J. B. W. Kok, G. Westendorp, "Optimizing a Steam Methane Reformer for Hydrogen Production,” International Journal of Hydrogen Production, vol. 34, pp. 285-292, 2009.

[4] M. W. Abdulrahman, "Experimental studies of direct contact heat transfer in a slurry bubble column at high gas temperature of a helium-water-alumina system, Applied Thermal Engineering, vol. 91, pp. 515-524, 2015.

[5] M. W. Abdulrahman, "Experimental studies of gas holdup in a slurry bubble column at high gas temperature of a helium- water- alumina system," Chemical Engineering Research and Design, vol. 109, pp. 486-494, 2016.

[6] M. W. Abdulrahman, "Experimental studies of the transition velocity in a slurry bubble column at high gas temperature of a helium-water-alumina system," Experimental Thermal and Fluid Science, vol. 74, pp. 404-410, 2016.

[7] M. W. Abdulrahman, "Direct contact heat transfer in the thermolysis reactor of hydrogen production cu-cl cycle," US Patent 10, 059, 586, August 28, 2018.

[8] M. W. Abdulrahman, "Analysis of the thermal hydraulics of a multiphase oxygen production reactor in the $\mathrm{Cu}-\mathrm{Cl}$ cycle,” Ph.D. dissertation, Dept. Mech. Eng., UOIT, Oshawa, ON., Canada, 2016.

[9] K. Bert, Heat and mass transport in tubular packed bed reactors at reacting and non- reacting conditions, Twente University Press, 2002.

[10] M. W. Abdulrahman, "CFD simulations of direct contact volumetric heat transfer coefficient in a slurry bubble column at a high gas temperature of a helium-water-alumina system, Applied Thermal Engineering, vol. 99, pp. 224234, 2016.

[11] M. W. Abdulrahman, "CFD Analysis of Temperature Distributions in a Slurry Bubble Column with Direct Contact Heat Transfer," in proceedings of the 3rd International Conference on Fluid Flow, Heat and Mass Transfer (FFHMT'16), Ottawa, ON, 2016. 
[12] M. W. Abdulrahman, "Heat Transfer in a Tubular Reforming Catalyst Bed: Analytical Modelling," in proceedings of the $6^{\text {th }}$ International Conference on Fluid Flow, Heat and Mass Transfer (FFHMT'19), Ottawa, ON, 2019.

[13] I. H. Sneddon, The Use of Integral Transforms, New York: McGraw-Hill, 1972.

[14] M. Van Genuchten and W. J. Alves, "Analytical solutions of the one-dimensional convective-dispersive solute transport equation,” US Department of agriculture, technical bulletin no. 1661, p. 151, 1982.

[15] R. Bauer and E. U. Schlünder, "Effective radial thermal conductivity of packings in gas flow. Part I. convective transport coefficient," Int. Chem. Eng., vol. 18, p. 181, 1978.

[16] R. Bauer and E. U. Schlünder, "Effective radial thermal conductivity of packings in gas flow. Part II. Thermal conductivity of the packing fraction without gas flow," Int. Chem. Eng., vol. 18, p. 189, 1978.

[17] J. Votruba, J. Hlavacek and M. Marek, "Packed bed axial thermal conductivity," Chem. Eng. Sci., vol. 27, p. 1845, 1972.

[18] R. A. Colledge, and W. R. Paterson, Coll. Papers, Instn. Chem. Engrs. 11 th Annual Res. Meeting, Bath, 1984.

[19] USAMI Yutaka, FUKUSAKO shoichiro and YAMADA Masahiko, "Heat and Mass Transfer in a Reforming Catalyst Bed: Quantitative Evaluation of the Controlling Factor by Experiment," Journal of Thermal Science and Technology, vol. 2, no. 2, 2007.

[20] Wen Dongsheng and Ding Yulong, "Heat Transfer of Gas Flow Through a Packed Bed", Chem. Eng. Sci., vol. 61, pp. 3532-3542, 2006. 